\title{
Comparison of Influence of the Fast Atom Beam and Ion Beam on the Metal Target
}

\author{
A. Pushkarev $(D$, A. Prima $(D$, , V. Myshkin $(D$, N. Chistyakova, and V. Ezhov \\ Tomsk Polytechnic University, 634050 Tomsk, Russia \\ Correspondence should be addressed to A. Pushkarev; aipush@mail.ru
}

Received 28 August 2020; Accepted 21 December 2020; Published 13 January 2021

Academic Editor: Dieter Hoffman

Copyright (C) 2021 A. Pushkarev et al. This is an open access article distributed under the Creative Commons Attribution License, which permits unrestricted use, distribution, and reproduction in any medium, provided the original work is properly cited.

\begin{abstract}
A comparative analysis of a fast atom beam and ion beam effect on a metal target in the binary collision model is performed. Irradiation by fast atoms has been shown to more closely correspond to neutron radiation in a nuclear reactor, in terms of the primary knocked-on atom spectrum and the efficiency and mechanism of the radiation defect formation. It was found that upon irradiation by fast carbon atoms with an energy of $0.2-0.3 \mathrm{MeV}$, the average number of radiation defects in the displacement cascade of one atom is four to five times higher than the calculated values using the SRIM program for ions with the same energy. It is shown that during penetration in the target, the probability of ionization of atoms with energies less than $0.4 \mathrm{MeV}$ is negligible.
\end{abstract}

\section{Introduction}

Charged-particle beams (electrons, ions, and plasma compression flow) are widely used in various technological processes and in the study of samples from structural materials. Thermal quenching (hardening) and hammering of metal products are the oldest methods employed to improve their operational properties. Improving the technological process of quenching can be achieved by increasing the product temperature change rates and processing only the surface layer, without changing the bulk properties. For this purpose, laser radiation [1], electron beam [2, 3], ion beam [4], and plasma compression beam [5] are used. Exposure of a high-intensity pulsed ion beam (HIPIB), with a duration of $100-150 \mathrm{~ns}$ and energy density $2-3 \mathrm{~J} / \mathrm{cm}^{2}$, provides heating and cooling of the processed product surface layer at $\sim 10^{8} \mathrm{~K} / \mathrm{s}$ [6]. When the HIPIB energy density is higher than $3 \mathrm{~J} / \mathrm{cm}^{2}$, ablation of the target material begins, forming recoil shock waves in the processed product, and the pressure exceeds $10^{8} \mathrm{~Pa}[7,8]$. A fast atom beam has many advantages over the ion beam. The use of a powerful beam of fast atoms for the modification of materials reduces its scattering when irradiating dielectric or slightly conductive surfaces [9]. Neutral beams are used to heat plasma in fusion experiments $[10,11]$.

The development of the nuclear industry, active space exploration, and the study of thermonuclear fusion require the development of structural materials with high radiation resistance, first of all contribution to thermonuclear technology and science with particular emphasis on plasma-wall interactions [12]. During the operation of the nuclear reactor, neutrons are formed, creating radiation defects in the structural elements of the reactor and reducing their mechanical resistance. It is impossible to prevent defect formation, reducing radiation damage, and it is necessary to ensure maximum efficiency of the defect annealing and restoration of the original structure of materials.

The development of new materials with high radiation resistance requires a high-intensity radiation source. Irradiation of samples in a nuclear reactor requires a long amount of time to process the necessary neutron fluency and postreactor exposure of materials to reduce radiation activity. One of the most important changes neutron radiation causes in metals is swelling [13]. It takes about a year to achieve a regime of noticeable swelling during irradiation by neutrons in a nuclear reactor. This process 
is faster when irradiated by ions $[14,15]$. Therefore, in recent years, simulation methods for the formation of radiation defects have been actively developed. Simulation irradiation of structural materials is carried out using electron beams [16] or ion beams [14, 17].

However, simulated irradiation by charged-particle beams has significant disadvantages compared to neutron irradiation in a nuclear reactor. The energy spectrum of primarily knocked-out atoms (PKA) in the target with ion irradiation is significantly different from the spectrum with irradiation by neutrons [18]. Iron target has about $80 \%$ PKA with energies of $30-60 \mathrm{keV}$ after irradiation by neutrons with energies $0.5-5 \mathrm{MeV}$ in the nuclear reactor [19]. Formation of radiation defects by ions in metals occurs at low-angle scattering on target atoms, and more than $90 \%$ of PKA have energies less than $1 \mathrm{keV}$ [20]. The difference between simulation (by ion or electron irradiation) and nuclear reactor radiation processes does not make it possible to make accurate predictions of radiation resistance of new structural materials in a nuclear reactor. Simulated irradiation of structural materials with accelerated atoms can overcome these disadvantages, but such studies are practically absent. Simulated irradiation of structural materials with accelerated atoms can also simplify and accelerate the study of activation of construction components due to beam loss in heavy-ion accelerators [21] and studies of collective effects in the absorption of accelerated particles in the target [22]. Accelerated atoms, when absorbed into the target, have significantly lower electron losses than ions. This makes it possible to increase the yield of isotopes per accelerated particle by hundreds of times. The purpose of this study is to compare the effect of a fast atom beam and ion beam on the metal target.

\section{Calculation of the PKA Energy Spectrum}

The energy spectrum of the PKA in the target is the most important parameter, determining the spatial distribution of primary radiation defects, duration, and efficiency of their subsequent annealing. Energy spectra of PKA, when irradiating a metal target with neutrons or ions, considerably differ $[18,20]$. Experimental or calculated data of PKA spectra upon absorption of fast atoms in a metal target are absent.

The formation of radiation defects by ions occurs during small-angle scattering on target atoms due to the Coulomb interaction of their nuclei $[4,7]$. The nuclei radii $\left(3.6 \cdot 10^{-5} \AA\right.$ for $\mathrm{Fe}[23])$ are significantly less than the distance between atoms in a metal target (lattice constant for $\alpha$-Fe is $2.9 \AA$ ). Therefore, the penetration process of ions and neutrons in the target can be considered in the model of independent binary collisions $[13,14]$. After an elastic collision of two particles, a fast particle (mass $m_{1}$ ) and a target atom (mass $m_{2}$ ), the total momentum and total energy of the colliding particles are preserved. The energy obtained by the PKA after the collision is equal to $[13,14]$

$$
E_{\mathrm{PKA}}=E_{0} \frac{4 m_{1} \cdot m_{2}}{\left(m_{1}+m_{2}\right)^{2}}\left(1-\cos ^{2} \frac{\theta}{2}\right) \text {, }
$$

where $E_{0}$ is the initial energy of a fast particle (ion, neutron, or fast atom) and $\theta$ is the scattering angle of this fast particle.

The probability of ion scattering by target atoms is described by the Rutherford formula:

$$
f_{i}(\theta)=\left(\frac{4 Z_{1} \cdot Z_{2} \cdot e^{2}}{E_{0}}\right)^{2} \frac{1}{\sin ^{4}(\theta / 2)},
$$

where $Z_{1}$ and $Z_{2}$ describe the nuclei charges of an ion and PKA, respectively, and $e$ is an electron charge.

The energy spectrum of the PKA is determined by the efficiency of kinetic energy transfer from the fast particle to PKA, equal to the product PKA energy on the probability of scattering. The dependence of the energy transfer efficiency on PKA energy is extreme due to a decrease in PKA energy and an increase in the probability of scattering with an increase in the impact parameter (or decrease in $\theta$ ), as seen in equations (1) and (2). From equations (1) and (2), we obtain the energy transfer efficiency in the elastic collision of an ion with PKA that is equal to

$$
\begin{gathered}
F_{i}=f_{i} E_{\mathrm{PKA}}=\frac{K}{1-\cos \theta}, \\
\text { where } K=\frac{4 m_{1} \cdot m_{2}\left(4 Z_{1} \cdot Z_{2} \cdot e^{2}\right)^{2}}{E_{0}\left(m_{1}+m_{2}\right)^{2}} .
\end{gathered}
$$

Figure 1 shows the efficiency of energy transfer when different ions are penetrated in an iron target. The efficiency of kinetic energy transfer is normalized, and the integral on all PKA energies $(>1 \mathrm{eV})$ is equal to 1 .

In modern calculations $[17,18,20]$, the same results are obtained for the energy of the primary knocked-out atoms in the target when irradiated with ions-PKA energy after ion irradiation (with energy $0.2-0.4 \mathrm{MeV}$ ) is less than $1 \mathrm{keV}$. It makes no sense to consider more complex models of ion radiation effects on the target, which describe more correctly elastic collisions of the ion with the target atom in the energy range of less than $30-40 \mathrm{eV}$, when the energy is not enough to form defects.

The process of neutron penetration in the target can also be considered with binary collisions of solid balls [14, 15], whose radii are equal to the neutron radius $\left(3.6 \cdot 10^{-5} \AA \hat{)}\right)$ and nuclei radius of the target atom. Their dimensions are much smaller than the distance between atoms in the metal target. The probability of the neutron colliding with the target atom increases with the growth of the impact parameter and can be written as

$$
\begin{aligned}
& f_{n u}(p)=\frac{2 \pi p}{\int_{0}^{r_{1}+r_{2}} 2 \pi p d p}=\frac{p}{r_{1}+r_{2}}=\cos \frac{\theta}{2}, \text { at } p<r_{1}+r_{2}, \\
& f_{n u}(p)=0, \quad \text { at } p>r_{1}+r_{2},
\end{aligned}
$$

where $p$ is the impact parameter and $r_{1}$ and $r_{2}$ are the neutron and nucleus radii of the target atom, respectively.

From equations (1) and (4), we obtain that the energy transfer efficiency in the elastic collision of a neutron with a PKA is equal to 


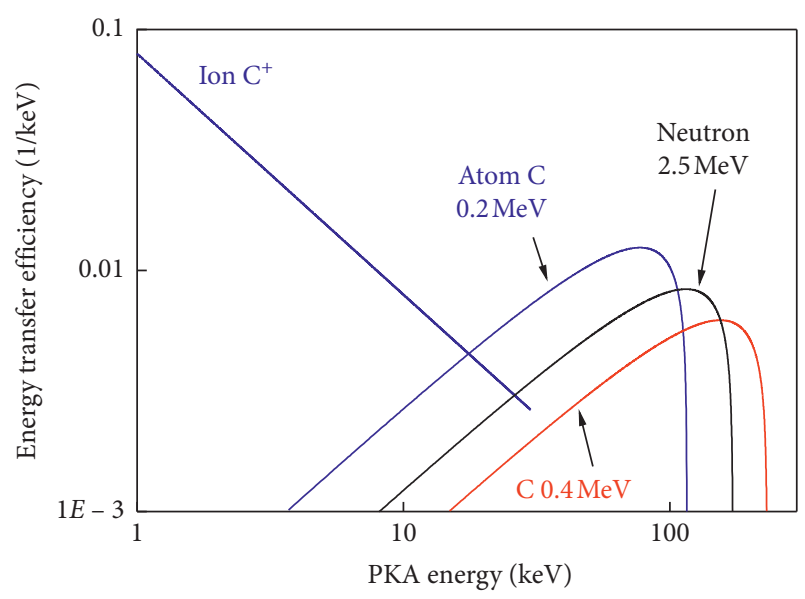

FIGURE 1: The dependence of the kinetic energy transfer efficiency from the PKA energy upon penetration in an iron target.

$$
\begin{aligned}
F_{n u} & =f_{n u} E_{\mathrm{PKA}} \\
& =E_{0} \frac{4 m_{1} \cdot m_{2}}{\left(m_{1}+m_{2}\right)^{2}}\left(1-\cos ^{2} \frac{\theta}{2}\right) \cdot \cos \frac{\theta}{2}, \quad \text { at } p<r_{1}+r_{2}, \\
F_{n u} & =0, \quad \text { at } p>r_{1}+r_{2} .
\end{aligned}
$$

Figure 1 shows the efficiency of energy transmission during neutron penetration in an iron target.

Despite numerous studies of the radiation processes, experimental and calculated data for the penetration of fast atoms in a metal target (PKA spectrum, energy balance, and depth of the penetration) are practically absent. The consideration of these processes in the ion deceleration model is incorrect, as the fast atoms with energy less than $100 \mathrm{keV}$ have an insignificant probability of ionization (see Section 5). A significant difference in the penetration mechanism of ions and fast atoms in the metal target is confirmed by the results of the energy balance study of the accelerated particle. Molecular dynamics (MD) modeling showed that PKA spends more than $60 \%$ of its energy on the formation of radiation defects in the target [24]. Calculations in SRIM showed that the electronic energy loss for a carbon ion with an energy of $200-250 \mathrm{keV}$ in various targets is 75-85\% [25].

A correct analysis of penetration of fast atoms in the iron target is possible in an MD simulation, setting a large energy to one of the target atoms (PKA method) at the beginning of the simulation. However, MD simulation requires large amounts of computation, which can only be implemented on supercomputers. When modeling the processes of a fast atom with an energy of $175 \mathrm{keV}$, an evolution analysis of $10^{6}-10^{7}$ atoms is needed [26]. The reliability of MD simulation largely depends on the choice of interatomic potential [27]; therefore, the simulation results are inconsistent. In addition, the use of the PKA method in MD simulation makes it possible to calculate the absorption of only the fast target atom.
Numerous experimental studies confirm the correctness of modeling the distribution of the ions over the depth of the target by binary collisions [19]. However, when simulating penetration of the fast atom in a metal target by this method, it is necessary to consider the elastic collision of balls with diameters equal to the atom diameter, not nuclei diameter. The radius of the iron atom is $1.56 \AA$ [23], and lattice constant for $\alpha$-Fe is $2.9 \AA$. Therefore, to calculate the PKA spectrum when irradiating the target with fast atoms, first consider the simplest option: penetration in the environment of atomic gas. Even with atmospheric pressure, the average distance between atoms exceeds the sizes of the atoms by approximately 10 times, and for the process of collision, it is possible to consider a binary collision model. The probability of the fast atom colliding with the target atom increases with the growth of the impact parameter and can be written as

$$
f_{a}(p)=\frac{2 \pi p}{\int_{0}^{R_{1}+R_{2}} 2 \pi p d p}=\frac{p}{R_{1}+R_{2}}=\cos \frac{\theta}{2}, \quad \text { at } p<R_{1}+R_{2} \text {, }
$$

where $R_{1}$ and $R_{2}$ are the radii of PKA and the fast atom, respectively.

The Lennard-Jones potential can be used to describe the interatomic interaction of a fast atom with an atomic gas $[27,28]$ :

$$
U(r)=4 \varepsilon\left[\left(\frac{R}{r}\right)^{-12}-\left(\frac{R}{r}\right)^{6}\right]
$$

where $\varepsilon$ describes the interatomic interaction force in the lattice (depth parameter) and $r$ is the distance to an atom.

For a fast atom with energy that is significantly higher than thermal energy, one can neglect the second stage, which describes the weak attraction caused by Van der Waals forces. The Lennard-Jones potential sharply decreases with increasing distance between atoms, and at a distance of $r=2.3 \mathrm{R}$, it is $0.016 \varepsilon$. Therefore, the probability of collision can be written as

$$
\begin{aligned}
& f_{a}(p)=\frac{p}{R_{1}+R_{2}}=\cos \frac{\theta}{2}, \quad \text { at } p<R_{1}+R_{2}, \\
& f_{a}(p)=\left(\cos \frac{\theta}{2}\right)^{-12}, \quad \text { at } p>R_{1}+R_{2} .
\end{aligned}
$$

Figure 2 shows the dependence of the scattering probability of the carbon atom on an iron atom on the impact parameter. For, a carbon atom, $R=0.67 \AA$, and for an iron atom, $R=1.56 \AA$. Dependence in Figure 2 is normalized, and the scattering probability integral for all possible values of the impact parameter is equal to one.

From equations (1) and (8), efficiency of kinetic energy transfer in elastic collision of a fast atom with an atomic gas atom is equal to 


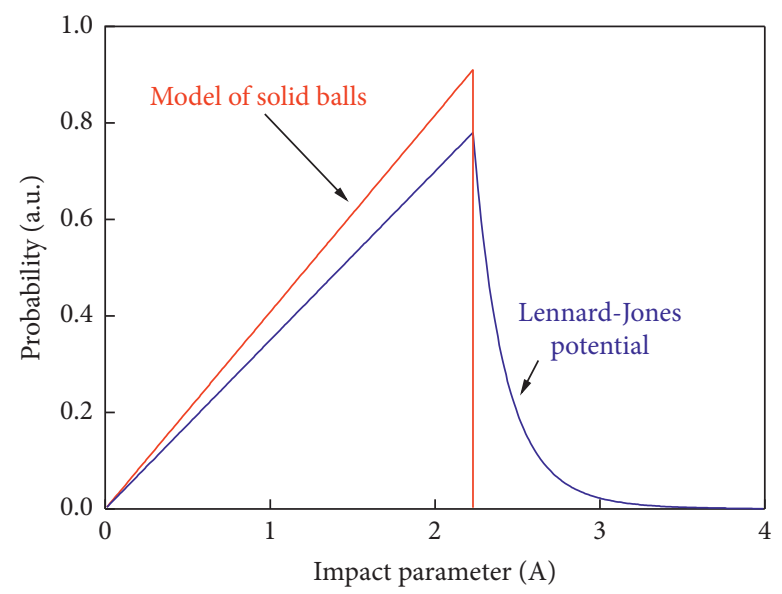

FIGURE 2: The dependence of scattering probability of the fast carbon atom on the iron atom on the impact parameter in the model of solid balls and taking into account the Lennard-Jones potential.

$$
\begin{aligned}
F_{a}= & f_{a}(p) E_{\mathrm{PKA}}=E_{0} \frac{4 m_{1} \cdot m_{2}}{\left(m_{1}+m_{2}\right)^{2}}\left(1-\cos ^{2} \frac{\theta}{2}\right) \\
& \cdot \cos \frac{\theta}{2}, \quad \text { at } p<R_{1}+R_{2}, \\
F_{a}= & f_{a}(p) E_{\mathrm{PKA}}=E_{0} \frac{4 m_{1} \cdot m_{2}}{\left(m_{1}+m_{2}\right)^{2}}\left(1-\cos ^{2} \frac{\theta}{2}\right) \\
& \cdot\left(\cos \frac{\theta}{2}\right)^{-12}, \quad \text { at } p>R_{1}+R_{2} .
\end{aligned}
$$

Figure 1 shows the efficiency of energy transfer upon penetration of a fast carbon atom in an atomic gas medium.

For a fast atom, the minimum scattering angle in the metal target corresponds to an impact parameter equal to half the distance between the target atoms:

$$
\cos \frac{\theta_{\min }}{2}=\frac{R_{1}}{R_{1}+R_{2}} \approx 0.7 \text {. }
$$

For a fast carbon atom and iron target, $\theta_{\min }=91^{\circ}$. Figure 3 shows the efficiency of energy transfer upon penetration of the fast carbon atom in an iron target.

The analysis showed that the PKA energy in the iron target, after irradiation by fast atoms (with energy $0.2-0.4 \mathrm{MeV}$ ) and neutrons (with energy $1-3 \mathrm{MeV}$ ), differs slightly and exceeds $50 \mathrm{keV}$. PKA energy after ion irradiation (with energy $0.2-0.4 \mathrm{MeV}$ ) is less than $1 \mathrm{keV}$. The PKA energy spectra, upon penetration of accelerated atoms in a solid target and gas, differ only in the low-energy area.

\section{Analysis of the Spatial Distribution of Radiation Defects}

The analysis of the spatial distribution of radiation defects in the target confirms the correctness of the PKA energy spectrum calculations when irradiating the target by fast atoms. The MD simulation shows [29] that when the PKA energy is higher than the critical value of $10 \mathrm{keV}$ (corresponding to neutron irradiation), subcascades are formed in the iron target, which indicates the transfer of a large amount of energy in elastic collisions with the target atoms. Irradiation of materials by electrons or ions leads to the appearance of mostly isolated interstitial atoms, vacancies, and small clusters of these point defects due to low PKA energy (0.1-1 keV, see Figure 1).

The generation mode of radiation defects in the metal target upon irradiation by fast atoms differs significantly from the mode upon irradiation by ions and corresponds more to the mode upon irradiation by neutrons. More than $90 \%$ of the PKA in the target after irradiation by fast atoms (with energy $0.2-0.4 \mathrm{MeV}$ ) have energies of more than $50 \mathrm{keV}$ (Figure 3). These PKA will form subcascades, rather than isolated interstitial atoms and vacancies.

The correctness of the PKA energy spectrum calculations, when irradiating the target with accelerated atoms, is also confirmed by the analysis of the scattering angle of the accelerated carbon atom, which is $90-120^{\circ}$ during penetration in an iron target (Figure 4).

The MD simulation also confirms that the PKA scattering angle is higher than $90^{\circ}$ (Figure 5) [26], and the formation of subcascades is perpendicular to the direction of the PKA movement.

The fast atom and PKA scattering in the target occurs mainly at a high angle, which provides a weak connection between penetration in the target and the fast atom energy. In low-angle ion scattering in a metal target, penetration is proportional to energy, allowing the term "linear energy loss" to be used when considering the elastic and nonelastic interactions of the ion with the target [24].

\section{Investigation of the Number of Radiation Defects}

In implantation and thermal modification of the metal sample surface layer, ions and fast atoms with the same energy will have the same effect. However, the efficiency of the radiation defect formation by fast atoms is higher than for ions with the same kinetic energy, as the energy losses by 


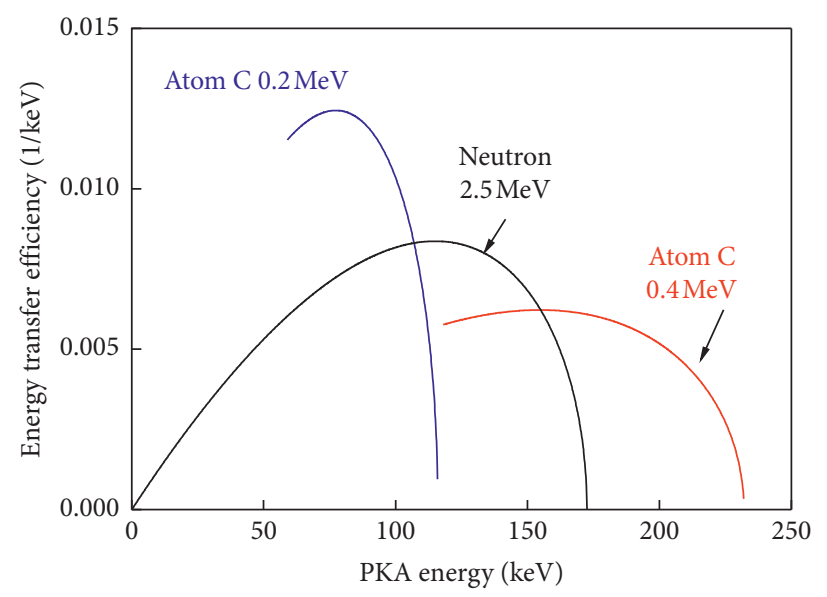

FIGURE 3: The dependence of the kinetic energy transfer efficiency of accelerated particles from the PKA energy upon penetration in an iron target.

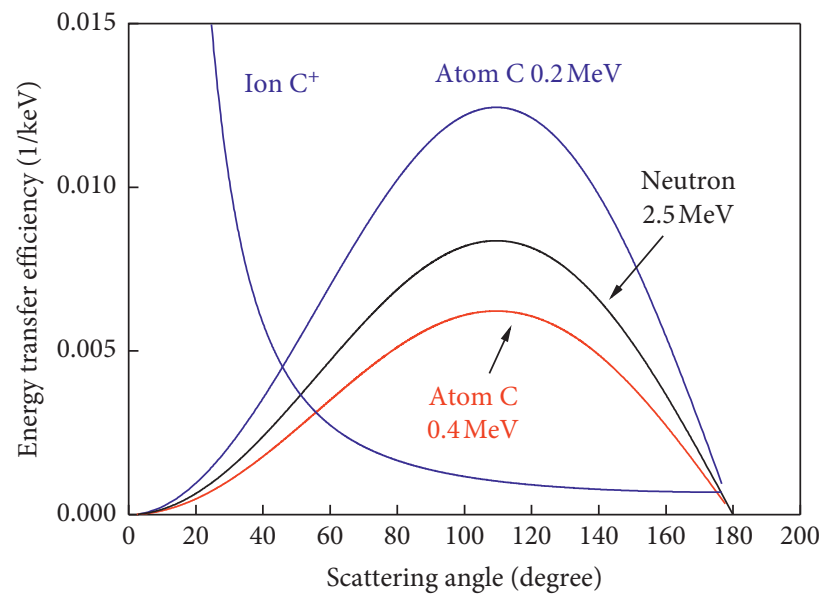

Figure 4: Dependence of energy transfer efficiency of accelerated particles on scattering angle in the iron target.

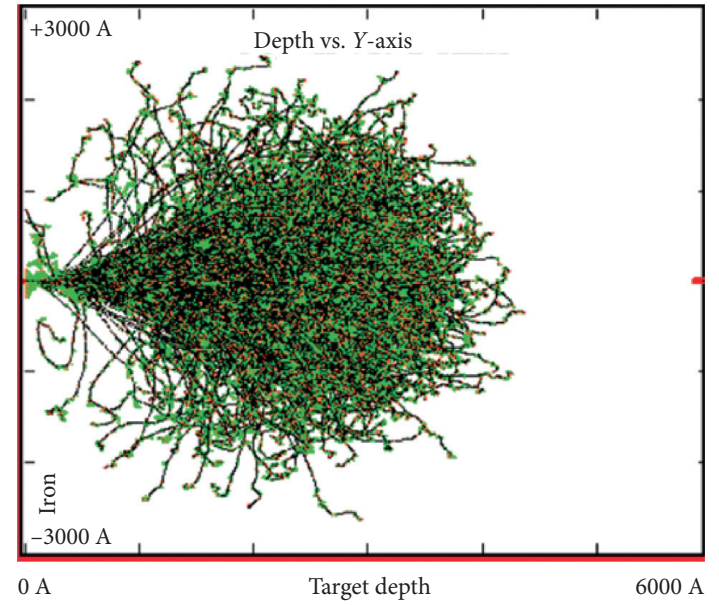

(a)

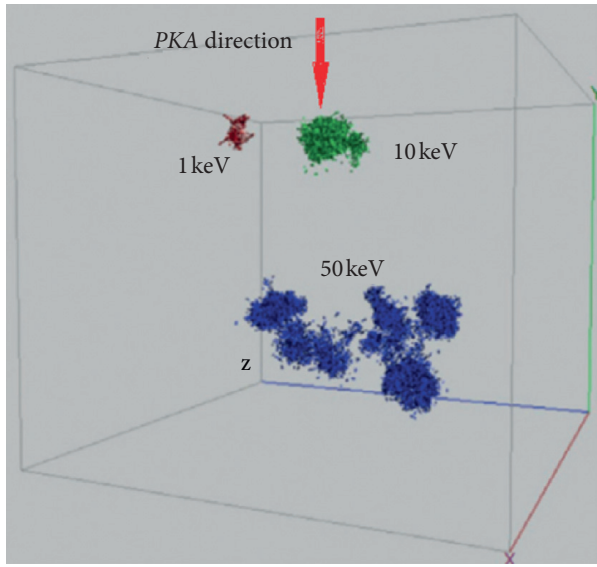

(b)

FIGURE 5: SRIM simulation of $\mathrm{C}^{+}$ions with $250 \mathrm{keV}$ energy in an iron target (a) and MD simulations of PKA displacement cascades in iron (b) [26]. 
fast atoms are less. An MD simulation has shown that the PKA consumes more than $60 \%$ of its energy to form radiation defects in the target [30]. When irradiating a metal target by ions, the main part of their kinetic energy is spent on the electronic stopping, without the formation of radiation defects $[14,15]$. Calculation in SRIM [31, 32] has shown that the electronic energy loss for a carbon ion with an energy of $200-250 \mathrm{keV}$ in various targets is $75-85 \%$. Therefore, we performed experimental studies of radiation defects upon irradiation of a metal target by a fast atom pulsed beam.

The experiments were carried out using a TEMP-6 accelerator (accelerating voltage $250-300 \mathrm{kV}$ and pulse duration $150 \mathrm{~ns}$ ) [33], consisting of a Marx generator, a pulsed forming line, and a vacuum ion diode with selfmagnetic insulation of electrons. The metal mesh in the field of ion transportation increased the charge exchange between the ions and residual gas molecules in the gas layer of desorbed molecules adjacent to the mesh, and the energy of fast atoms, in the HIPIB total energy, increased from 20 to $98 \%$ [34]. For analysis of the HIPIB parameters, we used thermal imaging diagnostics of the energy density (spatial resolution of $1-2 \mathrm{~mm}$ ) $[35,36]$ and time-of-flight diagnostics of the beam composition (time resolution of $1 \mathrm{ns)}$ [37].

These experiments were performed on a target with dimensions that were much smaller than the transverse dimensions of the fast atom beam. This ensured uniform irradiation and prevented the loss of thermal energy from the heated area due to thermal conductivity. A disc made of stainless steel foil, $0.1 \mathrm{~mm}$ thick and $10 \mathrm{~mm}$ in diameter, was installed in the central hole of a large stainless steel foil and was fixed with a thin stainless steel wire, with a $0.1 \mathrm{~mm}$ diameter.

The number of radiation defects was determined using calorimetric diagnostics, which is based on the comparison of the experimental power of energy loss in the target and the calculated power of thermal radiation when the target is cooled after irradiation by a fast atom beam or ions [25]. The number of radiation defects (vacancies + interstitial atoms) generated in the target was calculated from the following ratio:

$$
N_{d}=\frac{2 E_{a n}}{E_{d}},
$$

where $E_{a n}$ is the energy of radiation defect annihilation in the target after irradiation and $E_{d}$ is the threshold energy for radiation defect formation.

Our studies have shown that radiation defects can be divided into two groups: fast defects that are annihilated before the target cooling measurement (within $0.1 \mathrm{~s}$ after irradiation for $150 \mathrm{~ns}$ ) and slow defects that migrate to the target from their formation point and are, then, annihilated over tens of seconds [38]. Figure 6 shows the dependence of the number of radiation defects in the target on the absorbed beam energy [38]. We calculated the number of radiation defects for a threshold energy of $40 \mathrm{eV}$ for the iron target [39].

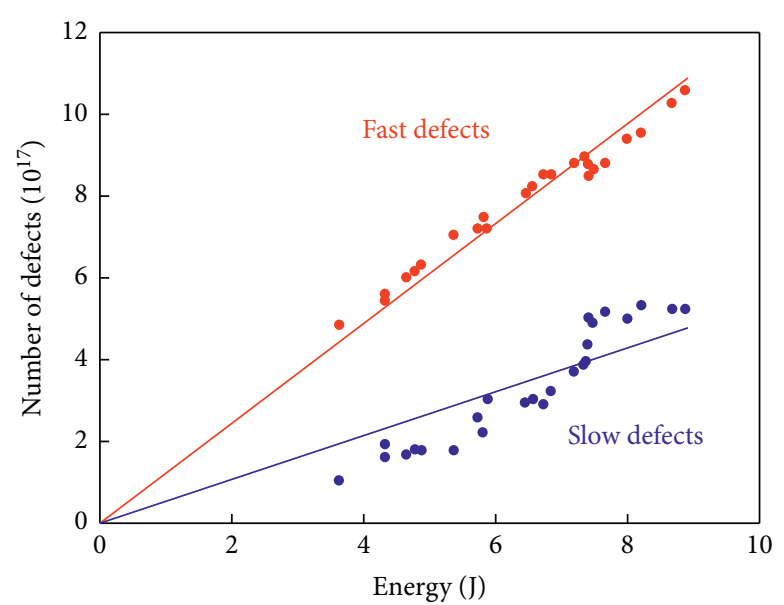

Figure 6: Dependence of the number of fast and slow defects on the absorbed beam energy in a stainless steel target.

Our research has shown that the dependence of the number of radiation defects in the target on the absorbed energy of the beam is described by a linear function (Figure 6): $N_{d}=K_{d} \cdot E_{\text {sum }}$, where $K_{d}$ is the coefficient equal to the number of radiation defects formed in the target by an ion beam with an energy of $1 \mathrm{~J}$.

One of the main parameters, calculated when modeling the formation of radiation defects in the target, is the number of defects in the cascade formed by a single accelerated particle. The average number of radiation defects in the displacement cascade can be calculated by the following ratio:

$$
n_{d}=K_{d} \cdot E_{\text {atom }}
$$

where $E_{\text {atom }}$ is the average energy of a fast atom in the beam. Table 1 shows the calculation of the number of defects in the fast carbon atom cascade.

In addition to experimental studies on the formation of radiation defects during irradiation of a metal target, we performed a simulation with SRIM. In the SRIM simulation, we calculated the total number of vacancies and interstitial atoms produced in the ion $\mathrm{C}^{+}$cascade with a lattice binding energy of $3 \mathrm{eV}$ and a threshold energy of $40 \mathrm{eV}$. The SRIM simulation results for an ion energy of $200 \mathrm{keV}$ are shown in Table 1 . The simulation showed that the number of radiation defects in the cascade of $\mathrm{C}^{+}$ions $\left(N_{\text {SRIM }}\right)$ is four to five times lower than the experimental values $\left(N_{\text {sum }}\right)$. These results confirm higher efficiency of radiation defect formation by fast atoms compared to ions.

\section{Fast Atom Ionization upon Absorption in the Target}

The analysis showed that irradiation of a metal target by fast atoms is more consistent with radiation by neutrons in a nuclear reactor, in terms of the PKA spectrum, efficiency, and mechanism of radiation defect formation. However, unlike neutrons, fast atoms can be ionized during penetration in a target; the energy required to remove an electron 
TABLe 1: Number of defects in the fast carbon atom cascade.

\begin{tabular}{lccc}
\hline Fast defects & Slow defects & $N_{\text {sum }}$ & $N_{\text {SRIM }}$ \\
\hline 4500 & 2025 & 6525 & 1250 \\
\hline
\end{tabular}

does not exceed $30 \mathrm{eV}$ and is significantly less than its kinetic energy.

The ion charge exchange was discovered by Henderson through experiments in which $\alpha$ particles $(5.6 \mathrm{MeV}, \alpha$-radon decay) passed through foil of mica or gold [40]. It was noted that after the passage of the foil, singly charged $\mathrm{He}^{+}$ions and neutral $\mathrm{He}$ atoms appeared, which was explained by the capture of electrons by $\alpha$ particles in the target. The MD simulation indicated absence confirmation of fast atom ionization during absorption of ions and neutrons in the metal target [29]. At PKA energies less than $200 \mathrm{keV}$, atoms, not ions, form radiation defects in the iron target.

The processes of elastic collisions, stripping (increasing the multiplicity of ionization), and charge exchange of an ion with target atoms are described in the binary collision approximation model $[13,14]$. It was shown in [41] that at a high ion energy and with an increase in the density of the medium, the charge exchange cross section decreases by $10-15 \%$, and the ionization cross section increases, since the incident ion is ionized, not only from the equilibrium but also from the excited states. Therefore, the processes of changing the charge state of ions and fast atoms during transportation in a solid and gaseous target will differ slightly.

The first research on changing the charge state of an ion during transportation in gas was carried out by Bohr [42]. It was found that when the ion velocity is less than the electron velocity in the orbit of the hydrogen atom $\left(2.2 \cdot 10^{8} \mathrm{~cm} / \mathrm{s}\right)$, the electron is captured (charge exchange). This condition corresponds to an ion energy less than $20.5 \mathrm{keV} /$ nucleon or $250 \mathrm{keV}$ for a carbon ion. The cross section for resonant proton charge exchange (with an energy of $20 \mathrm{keV} /$ nucleon) by atomic hydrogen is $5 \cdot 10^{-16} \mathrm{~cm}^{2}$, and the ionization cross section is much smaller, $6 \cdot 10^{-17} \mathrm{~cm}^{2}$ [43]. The monograph [44] presents the experimental and calculated cross-section values for the charge exchange and ionization of heavy ions upon absorption in gaseous and solid targets. When an iodine ion, with an energy less than $5 \mathrm{MeV}$, passes through hydrogen and oxygen, the cross section of capture of one electron is much larger than the ionization cross section. Tolstikhina and Shevelko [45] reviewed experimental data and theoretical methods for calculating the effective cross sections for charge exchange and ionization of multiply charged ions colliding with neutral atoms in a gas at energies up to $10 \mathrm{GeV} /$ nucleon. Such processes, with a change in the charge state, occur with high probability (total cross sections: $10^{-14}-10^{-16} \mathrm{~cm}^{2}$ ). It was shown that at an ion energy less than $10 \mathrm{MeV} /$ nucleon, charge exchange processes mainly occur with a decrease in the ionization rate. Fast carbon atoms obtained at the TEMP-6 accelerator have an energy of $250-300 \mathrm{keV}$, and upon absorption in the target, charge exchange, rather than ionization, is more likely.

The results of an experimental study of the fast atom generation in an ion diode with self-magnetic insulation of electrons are presented in our article [46]. The HIPIB energy density was measured using infrared imaging diagnostics and was calculated using the ion current density and accelerated voltage. The energy density from the infrared measurements exceeded measurements from the ion current density, indicating that fast atoms are present in the beam. Our studies have shown that the use of a metal mesh in the field of the HIPIB transportation increases the energy of fast atoms in the HIPIB total energy from $15-30$ to $98 \%$. The total energy of the combined beam (ions + fast atoms) does not change (considering the optical transparency of the mesh).

Fast atoms in an ion diode are produced through charge exchange between the ions and residential gas molecules in the gas layer adjusting the mesh. During desorption of molecules from the metal mesh surface, the thickness of the sheath adjusting the mesh exceeds, by $5-15$ times, the charge exchange length of the ions in the processes $\mathrm{C}^{+}+\mathrm{N}_{2} \longrightarrow \mathrm{C}^{0}$ and $\mathrm{C}^{+}+\mathrm{O}_{2} \longrightarrow \mathrm{C}^{0}[47-49]$. When generating $\mathrm{N}^{2+}$ ions, the use of a metal mesh in the transport region ensures recharging of $\mathrm{N}^{2+} \longrightarrow \mathrm{N}^{+}$ions.

\section{Conclusion}

The performed analysis showed that pulsed beams of fast atoms are most effective in simulated radiation exposure of structural materials. Irradiation of a metal target by fast atoms is more consistent with neutron irradiation in a nuclear reactor, according to the PKA spectrum, efficiency, and radiation defect formation mechanism. The PKA energy in the target, irradiated with fast atoms (with an energy of $200 \mathrm{keV}$ ) and neutrons (with an energy of $3 \mathrm{MeV}$ ), exceeds $10 \mathrm{keV}$, and the PKA energy after ion irradiation (with an energy of $200 \mathrm{keV}$ ) is less than $100 \mathrm{eV}$. The computer simulation showed that the number of radiation defects in the cascade of $\mathrm{C}^{+}$ions is four to five times lower than the experimental values in the cascade of carbon atom with the same energy.

The PKA energy spectrum upon irradiation of a metal target by fast atoms, with an energy of $200-600 \mathrm{keV}$, more fully corresponds to the PKA spectrum upon irradiation by neutrons formed in the fission reaction ${ }^{235} \mathrm{U}$ in a nuclear reactor for targets made of titanium, copper, zirconium, etc. Irradiation with a beam of fast atoms makes it possible to study the radiation resistance of dielectric and weakly conducting materials, which significantly expands the scope of the installation.

Unlike a neutron, an accelerated atom can be ionized during absorption in a target; the energy required to remove an electron is much less than its kinetic energy. However, at the energy of fast atoms, $200-600 \mathrm{keV}$, the ion charge exchange cross section during penetration in the target is significantly higher than the ionization cross section, and the ionization probability is negligible. High efficiency of ion charge exchange during the fast atom pulsed beam formation in the diode with passive anode, MD simulation of the displacement cascade formation during the penetration of ions and neutrons in the target, and formation of singly charged $\mathrm{He}^{+}$ions and neutral helium atoms when $\alpha$ particles pass through the foil confirm the low probability of ionization of accelerated atoms during absorption in the target. 


\section{Data Availability}

Hyperlinks to publicly archived datasets analyzed or generated during the study are listed in the references.

\section{Conflicts of Interest}

The authors declare that they have no conflicts of interest.

\section{Authors' Contributions}

All authors contributed equally to this work.

\section{Acknowledgments}

This study was supported by the Russian Foundation for Basic Research, project no. 19-38-90001.

\section{References}

[1] S. Kano and T. Inoue, "Surface softening and hardening of WC-Co using pulsed laser irradiation," Surface and Coatings Technology, vol. 201, no. 1-2, pp. 223-229, 2006.

[2] V. Rotshtein, Y. Ivanov, and A. Markov, "Surface treatment of materials with low-energy, high-current electron beams," Materials surface Processing by Directed Energy Techniques, European Materials Research Society, Strasbourg, France, pp. 205-240, 2006.

[3] D. I. Proskurovsky, V. P. Rotshtein, G. T. Ozur, and Yu.F. Ivanov, "Markov Physical foundations for surface treatment of materials with low energy, high current electron beams," Surface and Coatings Technology, vol. 125, no. 1-3, pp. 49-56, 2000.

[4] M. Nastasi, J. W. Mayer, and Y. Wang, Ion Beam Analysis: Fundamentals and Applications, CRC Press, Boca Raton, FL, USA, 2014.

[5] V. V. Uglov, V. M. Anishchik, V. M. Astashynski, N. N. Cherenda, I. G. Gimro, and A. V. Kovyazo, "Modification of WC hard alloy by compressive plasma flow," Surface and Coatings Technology, vol. 200, no. 1-4, pp. 245-249, 2005.

[6] Yu Xiao, J. Shen, M. Qu et al., "Distribution and evolution of thermal field formed by intense pulsed ion beam on thin metal target," Nuclear Instruments and Methods in Physics Research Section B: Beam Interactions with Materials and Atoms, vol. 365, pp. 225-229, 2015.

[7] V. I. Boyko, V. A. Skvortsov, V. E. Fortov, and I. V. Shamanin, The Charged Particles Pulsed Beams Interaction with Matter, Fizmatlit, Moscow, Russia, in Russian, 2003.

[8] A. I. Pushkarev, Y. I. Isakova, Y. Xiao, and I. P. Khailov, "Characterization of intense ion beam energy density and beam induced pressure on the target with acoustic diagnostics," Review of Scientific Instruments, vol. 84, Article ID 083304, 2013.

[9] S. N. Grigoriev, Y. A. Melnik, A. S. Metel, and M. A. Volosova, "Focused beams of fast neutral atoms in glow discharge plasma," Journal of Applied Physics, vol. 121, no. 22, Article ID 223302, 2017.

[10] H. Horiike, M. Akiba, M. Araki et al., "Extraction electrode for a $100-\mathrm{kV}, 40-\mathrm{A}, 10$-s ion source," Fusion Technology, vol. 7, no. 2, pp. 171-179, 1985.

[11] U. Fantz, P. Franzen, W. K. M. Berger et al., "Negative ion RF sources for ITER NBI: status of the development and recent achievements plasma," Physics and Controlled Fusion, vol. 49, no. 12B, p. B563, 2007.
[12] M. Rubel, S. Brezinsek, J. W. Coenen et al., "Overview of wall probes for erosion and deposition studies in the TEXTOR tokamak," Matter and Radiation at Extremes, vol. 2, no. 3, p. 87, 2017.

[13] K. Nordlund, S. J. Zinkle, A. E. Sand et al., "Primary radiation damage: a review of current understanding and models," Journal of Nuclear Materials, vol. 512, pp. 450-479, 2018.

[14] G. S. Was, Z. Jiao, E. Getto et al., "Emulation of reactor irradiation damage using ion beams," Scripta Materialia, vol. 88, pp. 33-36, 2014.

[15] C. Abromeit, "Aspects of simulation of neutron damage by ion irradiation," Journal of Nuclear Materials, vol. 216, pp. 78-96, 1994.

[16] Y. Satoh, Y. Abe, H. Abe et al., "Vacancy effects on onedimensional migration of interstitial clusters in iron under electron irradiation at low temperatures," Philosophical Magazine, vol. 96, no. 21, pp. 2219-2242, 2016.

[17] G. S. Was and R. S. Averback, "Radiation damage using ion beams," in Comprehensive Nuclear Materials, vol. 1, pp. 195-221, Elsevier, Amsterdam, Netherlands, 2012.

[18] G. S. Was, "Challenges to the use of ion irradiation for emulating reactor irradiation," Journal of Materials Research, vol. 30, no. 9, pp. 1158-1182, 2015.

[19] R. Smith, M. Jakas, D. Ashworth et al., "Atomic and ion collisions in solids and surfaces," Theory, Simulation and Application, p. 309, Cambridge University Press, Cambridge, UK, 2005.

[20] S. J. Zinkle and L. L. Snead, "Opportunities and limitations for ion beams in radiation effects studies: bridging critical gaps between charged particle and neutron irradiations," Scripta Materialia, vol. 143, pp. 154-160, 2018.

[21] P. Katrík, H. Dieter, H. Hoffmann, E. Mustafin, and I. Strašík, "Experimental study of residual activity induced in aluminum targets irradiated by high-energy heavy-ion beams: a comparison of experimental data and FLUKA simulations," Matter and Radiation at Extremes, vol. 4, no. 5, Article ID 055403, 2019.

[22] J. Ren, Z. Deng, W. Qi et al., "Observation of a high degree of stopping for laser-accelerated intense proton beams in dense ionized matter," Nature Communications, vol. 11, p. 5157, 2020.

[23] J. C. Slater, "Atomic radii in crystals," The Journal of Chemical Physics, vol. 41, no. 10, pp. 3199-3204, 1964.

[24] G. S. Was, Fundamentals of Radiation Materials Science. Metals and Alloys, Springer, Berlin, Germany, 2017.

[25] A. Pushkarev, A. Prima, X. P. Zhu et al., "Thermal imaging diagnostics of fast radiation processes," in Proceedings of the 6th International Congress on Energy Fluxes and Radiation Effects (EFRE 2018): Abstracts: Tomsk: Publishing House of IAO SB RAS, p. 516, Tomsk, Russia, September 2018.

[26] S. J. Zinkle, "Radiation-induced effects on microstructure," in Comprehensive Nuclear Materials, vol. 1, pp. 65-98, Elsevier, Amsterdam, Netherlands, 2012.

[27] G. J. Ackland, "Interatomic potential development," in Comprehensive Nuclear Materials, vol. 1, pp. 267-291, Elsevier, Amsterdam, Netherlands, 2012.

[28] J. E. Lennard-Jones, "On the determination of molecular fields," Proceedings of the Royal Society of London Series A, vol. 106, no. 738, pp. 463-477, 1924.

[29] W. Cai, J. Li, and S. Yip, "Molecular dynamics," in Comprehensive Nuclear Materials, vol. 1, pp. 249-265, Elsevier, Amsterdam, Netherlands, 2012.

[30] R. E. Stoller, "Primary radiation damage formation," Comprehensive Nuclear Materials, vol. 1, pp. 293-332, 2012. 
[31] J. F. Ziegler, J. P. Biersack, and U. Littmark, The Stopping and Range of Ions in Solids, Pergamon, New York, NY, USA, 1985.

[32] J. F. Ziegler, "SRIM \& TRIM," 2013, http://www.srim.org/.

[33] X. P. Zhu, M. K. Lei, and T. C. Ma, "Characterization of a high-intensity bipolar-mode pulsed ion source for surface modification of materials," Review of Scientific Instruments, vol. 73, no. 4, pp. 1728-1733, 2002.

[34] A. I. Prima, A. I. Pushkarev, C. Zhang, Y. Li, X. P. Zhu, and M. K. Lei, Laboratory Stand for Imitation Radiation Irradiation with Accelerated Atoms, XVI Kurchatov Interdisciplinary Youth Scientific School, Moscow, Russia, 2019.

[35] H. A. Davis, R. R. Bartsch, J. C. Olson, D. J. Rej, and W. J. Waganaar, "Intense ion beam optimization and characterization with infrared imaging," Journal of Applied Physics, vol. 82, no. 7, pp. 3223-3231, 1997.

[36] Y. I. Isakova and A. I. Pushkarev, "Visualization and analysis of pulsed ion beam energy density profile with infrared imaging," Infrared Physics \& Technology, vol. 89, pp. 140-146, 2018.

[37] A. I. Pushkarev, Y. I. Isakova, and A. I. Prima, "High-intensity pulsed ion beam composition and the energy spectrum using the time-of-flight method," Laser and Particle Beams, vol. 36, no. 2, pp. 210-218, 2018.

[38] A. Prima, R. Zyryanova, N. Pereday, and V. Medvedsky, "Determination of radiation defects quantity in metals under irradiation by heavy carbon ions," in Proceedings of the 24th International Conference on Ion-Surface Interactions, pp. 186-189, Moscow, Russia, August 2019.

[39] ASTM E521-16, Standard Practice for Investigating the Effects of Neutron Radiation Damage Using Charged-Particle Irradiation, ASTM International, West Conshohocken, PA, USA, 2016, http://www.astm.org.

[40] G. H. Henderson, "Changes in the charge of an $\alpha$-particle passing through matter," Proceedings of the Royal Society of London. Series A, vol. I02, pp. 496-506, 1923.

[41] I. Y. Tolstikhina and V. P. Shevelko, "Influence of atomic processes on charge states and fractions of fast heavy ions passing through gaseous, solid, and plasma targets," PhysicsUspekhi, vol. 61, no. 3, pp. 247-279, 2018.

[42] N. Bohr, The Penetration of Atomic Particles through Matter, Munksgaard, Copenhagen, Denmark, 1948.

[43] B. H. Bransden and M. R. C. Mcdowell, Charge Exchange and the Theory of Ion-Atom Collisions, Clarendon Press, Oxford, UK, 1992.

[44] H.-D. Betz, "Charge states and charge-changing cross sections of fast heavy ions penetrating through gaseous and solid media," Reviews of Modern Physics, vol. 44, no. 3, p. 465, 1972.

[45] I. Y. Tolstikhina and V. P. Shevelko, "Collision processes involving heavy many-electron ions interacting with neutral atoms," Physics-Uspekhi, vol. 56, no. 3, pp. 213-242, 2013.

[46] A. I. Pushkarev, Y. I. Isakova, and I. P. Khaylov, "Experimental evidence of energetic neutrals production in an ion diode," Nuclear Instruments and Methods in Physics Research Section B: Beam Interactions with Materials and Atoms, vol. 343, pp. 138-145, 2015.

[47] N. V. Novikov and Ya.A. Teplova, "Database on chargechanging cross sections in ion-atom collisions," Journal of Physics: Conference Series, vol. 194, Article ID 082032, 2009.

[48] L. M. Rottmann, R. Bruch, P. Neill, C. Drexler, R. D. DuBois, and L. H. Toburen, "Single-electron capture by 100-1500-keV $\mathrm{C}^{+}$ions in several atomic and molecular targets," Physical Review A, vol. 46, no. 7, pp. 3883-3888, 1992.

[49] I. T. Serenkov and P. I. Il'in, V. A. Oparin, “Solov'v," Journal of Experimental and Theoretical Physics, vol. 68, pp. 1686-1692, 1975. 